\title{
Robust Environmental Life Cycle Assessment of Electric VTOL Concepts for Urban Air Mobility
}

\author{
Nicolas André * and Manfred Hajek ${ }^{\dagger}$ \\ Institute of Helicopter Technology, \\ Technical University of Munich, Munich 80333, Germany
}

\begin{abstract}
Under which scenario is Urban Air Mobility more sustainable than ground-based mobility? To answer this question, we provide a Life Cycle Assessment of three electric Vertical Take-Off and Landing concept aircraft, including a quantification of uncertainties in the concept's material composition. We conduct a Cradle-to-Gate analysis of the concepts and extend it by a Wellto-Shaft analysis of Urban Air Mobility operation, including all relevant upstream greenhouse gas emissions due to battery use, again including input uncertainties. As for aviation systems in general, we show that the impact of power demand in operation is most significant and exceeds emissions from production by orders of magnitude. We thus provide sensitivity analyses of the results for each of the most influential quantities. We report an optimum flight speed for minimum greenhouse gas emissions and a quantification of the impact of hover flight. Finally, we compare and quantify the impact's sensitivities on influential factors like the region of operation, and mission design within reasonable ranges. From the sensitivity analyses, we conclude that only very lightweight vehicles for Urban Air Mobility can be more sustainable than traditional, fossil-fueled ground-based transportation, given a maximum seat utilization, clean power grid, low hover share and an effective reduction in travel distance. We further conclude that, with a combination of the most optimistic assumptions, Urban Air Mobility concepts may environmentally compete with battery-powered cars.
\end{abstract}

\section{Nomenclature}

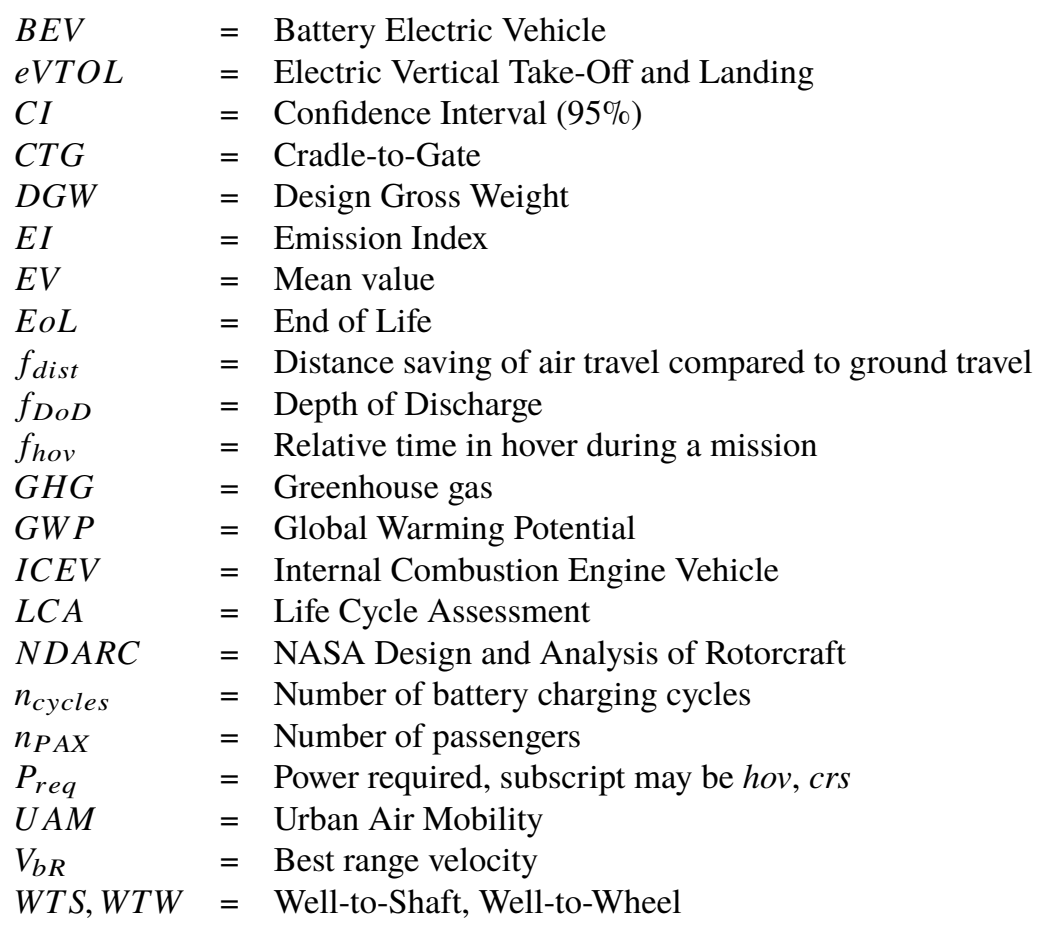

\footnotetext{
*Research Associate, PhD Candidate, nicolas.andre@tum.de

${ }^{\dagger}$ Head of the Institute, Professor, hajek@tum.de
} 


\section{Introduction}

Research, development, and construction of electric Vertical Take-Off and Landing (eVTOL) aircraft for Urban Air Mobility (UAM) are thriving [1, 2]. To date, more than 150 UAM concepts are being developed, covering a multitude of different configurations. To facilitate the technological advancement in UAM, NASA published mission and sizing studies on different feasible aircraft configurations with a variety of rotor configurations, wing attachments, payload, propulsion technology, and other quantities [3]. Of those studies, Silva et al. most recently presented concepts for a Quadrotor, a Side-by-Side Helicopter, and a Lift+Cruise aircraft, all capable of performing a reference UAM mission defined by Patterson et al. [4, 5]. Many studies line out the specific challenges regarding UAM, such as battery technology, autonomy, infrastructure, public acceptance and certification [6, 7]. Use cases such as the one presented by Uber, a transportation network company, suggest a dramatic reduction of travel time and cost, mostly based on the decreasing absolute transport distance, the avoidance of road congestion, and a lean infrastructure [8]. To date, it is however not clear whether eVTOL use in urban mobility is environmentally sustainable.

A study recently published in 'nature Communications' by Kasliwal et al. demonstrates that eVTOL may, in a constrained scenario, be able to environmentally compete with internal combustion engine vehicles, and possibly with battery electric vehicles [9]. The authors demonstrate the criticality of trip distance for the overall carbon efficiency and show that eVTOL can, in certain cases, beat ground-based cars in terms of emissions per passenger transported. They point out the importance of high seat utilization and area of operation as well as the influence of grid carbon intensity and battery capacity. We use this result as a starting point for our study, as we provide an analytic approach to quantify and visualize the impact of the listed factors on sustainability. We further include the influence of material production into our analysis by conducting a Life Cycle Assessment. Thereby, we can also assess the overall potential of the utilization of bio-based materials in a holistic life cycle context. Respective research by Strohrmann and Hajek recently showed a possible design of a bio-based helicopter tailplane [10].

The Life Cycle Assessment (LCA) methodology is a tool to comprehensively analyze the environmental impact of products or processes. It is well accepted in the scientific community and has already been applied to assess aircraft and helicopter configurations by Johanning and by Basset et al., respectively [11, 12]. However, major shortcomings of LCA are the limited availability of data, the inherent uncertainty in data and models, as well as variability of data, e.g. in the strong regional and temporal dependency of grid carbon intensity [13]. When analyzing a system at the stage of conceptual design, as we do with eVTOL concepts, the existing uncertainty is strongly amplified by a lack of knowledge on the design itself, the materials used and the required underlying processes.

To present a reliable answer to whether UAM may be more sustainable than ground-based mobility despite the outlined uncertainties, we provide a three-step approach: First, we conduct a Life Cycle Assessment of UAM, including statistical variations of uncertain quantities where possible and reasonable. In the LCA we focus on the impacts of material production and eVTOL operation by providing distinct Cradle-to-Gate (CTG) and Well-to-Shaft (WTS) analyses, respectively. We conclude the LCA by a comparison of the CTG and WTS analyses to quantitatively depict the dominant role of required power over once-in-a-life-cycle impacts in aviation. Second, we compare the three eVTOL concepts in terms of their specific operational greenhouse gas (GHG) impact, again including uncertainties in underlying data. With an analytical, quantitative analysis of the impacts sensitivities in the third step, we highlight the most influential factors on the sustainability of eVTOL and compare the calculated impacts with literature data for ground-based cars.

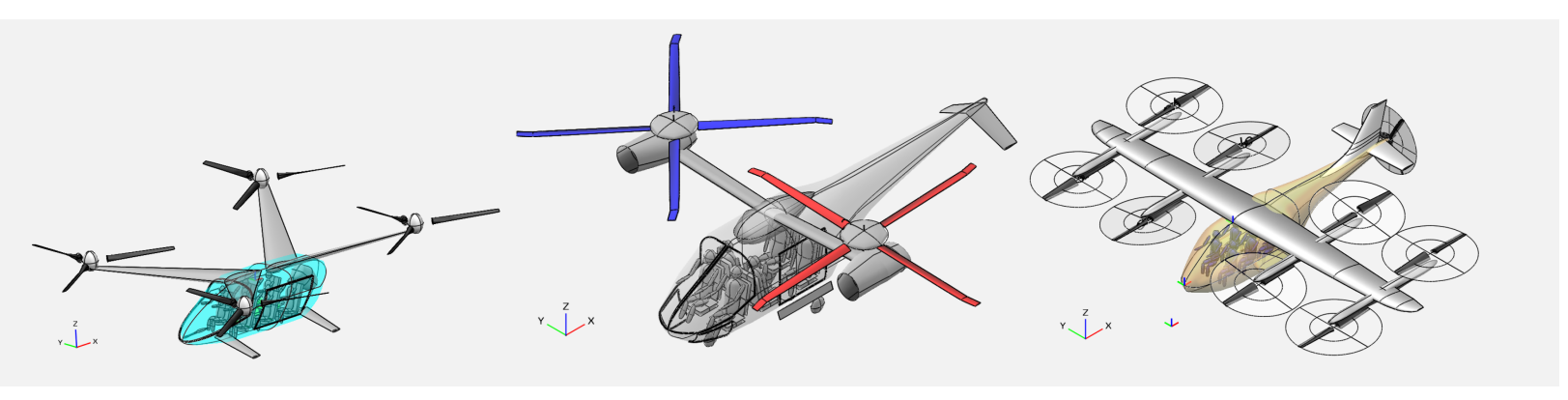

Fig. 1 Concept aircraft for Urban Air Mobility as published by Silva et al. [4] 


\section{Methodology}

\section{Concepts for UAM Reference Mission}

The three concept aircraft for UAM following the publications by Silva et al. and Johnson et al. are depicted in Figure 1. The Quadrotor (left), Side-by-Side (center) and Lift+Cruise (right) configurations each feature an electric and a fossil-fuel-powered version and are designed to transport a maximum payload of 6 passengers $(1200 \mathrm{lb})$ [3, 4]. The designs were computed with a sizing suite including NDARC, CAMRAD II and other tools developed by NASA for the analysis and conceptual design of rotorcraft [14]. For this study, we used the electric versions of the presented concepts as a basis. We recomputed the three concepts using NDARC, employing the same sizing mission and seed aircraft as provided by NASA. The concept's calculated sub-system masses are required as input for the LCA and are listed in Table 1. Table 1 also provides the concept's empty weight ratio, disk loading, and drag in forward flight, expressed by the equivalent flat plate area. As in the cited literature, we assumed the effective energy density of the Lithium-Ion battery on pack level to be $400 \mathrm{Wh} / \mathrm{kg}$ for all three cases. Further data on the concepts can be found in the respective works by Silva et al. and Johnson et al. [3, 4].

Table 1 Sub system masses and key attributes of the concept aircraft

\begin{tabular}{|c|c|c|c|c|c|c|c|}
\hline \multirow[b]{2}{*}{ Units } & EW/DGW & DGW & Structure \& Props & Engine \& Battery & Systems & \multirow{2}{*}{$\frac{\text { Disk Load. }}{\left[\mathrm{N} / \mathrm{m}^{2}\right]}$} & \multirow{2}{*}{$\begin{array}{l}\text { Drag } \\
{\left[\mathrm{m}^{2}\right]}\end{array}$} \\
\hline & {$[-]$} & \multicolumn{4}{|c|}{$[\mathrm{kg}]$} & & \\
\hline Quadrotor & 0.50 & 2939.2 & 744.5 & 1211.2 & 243.1 & 143.7 & 1.20 \\
\hline Side-by-Side & 0.49 & 2222.3 & 544.5 & 765.8 & 229.8 & 167.6 & 0.70 \\
\hline Lift+Cruise & 0.59 & 4309.8 & 786.1 & 1300.8 & 242.5 & 627.5 & 1.57 \\
\hline
\end{tabular}

\section{Life Cycle Assessment}

The Life Cycle Assessment (LCA) methodology is used to assess the environmental impact of products throughout all life cycle stages, from material extraction and production to end-of-life treatment. It has been subject to an ever-growing field of research since the 1990s and has found application in academia, industry, and policy-making [13]. LCA may cover a multitude of impact categories such as acidification, particulate matter formation, human toxicity, fossil depletion or freshwater eutrophication [15, 16]. For the present study, we confined the scope to the emission of greenhouse gases (GHG), expressed as Global Warming Potential (GWP). GWP measures the relative impact of GHG emission with respect to a given timeframe [15]. It is expressed as a mass equivalent of $\mathrm{CO}_{2}$ emissions. The timeframe we chose is 100 years, which is in line with scientific consensus [15]. We consider the limitation of our LCA to global warming reasonable, as it is one of the most pressing sustainability issues to date [17]. Also, this approach is in line with the referenced study by Kasliwal et al. [9].

\section{Impact computation including uncertainties}

The impact of the production phase stems from the extraction of raw materials and the production steps in the manufacturing process. Equation 1 describes the compilation of this impact in the Cradle-to-Gate (CTG) analysis of LCA. As outlined, we analyze the Global Warming Potential (GWP), but the Equations utilize the letters EI to denote emission indices in a more general fashion.

$$
E I_{C T G}=\sum_{i, j} E I_{i} \cdot f_{i, j} \cdot m_{j}
$$

The total emission index of the production of a system is calculated as the sum of emission indices $E I_{i}$ of used materials $i$ in the system's components $j$. The component masses and material fractions in the components are thus denoted as $m_{j}$ and $f_{i, j}$, respectively. The components of the eVTOL concepts and their masses are listed in Table 1 . The total material fractions of the eVTOL concepts, i. e. summed up over the $j$ components, are listed in Table 2 This material composition is compiled based on the sub-system masses of the NDARC models and the assumed sub-system material fraction. Table 2 also shows the ranges of respective GWP emission indices per material, which are taken from the referenced literature. Obviously, there are large variations in the GWP emission indices, e.g. for the production of 
Table 2 Assumed material composition of the concepts and GWP emission indices of materials. References for emission indices are given by [19-22]

\begin{tabular}{lccccccc}
\hline & Aluminium & Steel & Titanium & Copper & Carbon Fiber & Glass Fiber & Polymers \\
\hline Quadrotor & 0.158 & 0.103 & 0.076 & 0.128 & 0.192 & 0.125 & 0.222 \\
Side-by-Side & 0.147 & 0.079 & 0.056 & 0.159 & 0.250 & 0.101 & 0.218 \\
Lift+Cruise & 0.127 & 0.082 & 0.050 & 0.107 & 0.263 & 0.128 & 0.172 \\
\hline GWP $\left[\frac{\mathrm{kg} \mathrm{CO} \text { eq }}{\mathrm{kg}}\right]$ & $8.3-27.7$ & $1.9-3.0$ & $31.3-49.0$ & $1.3-2.3$ & $28.5-35.2$ & $7.5-8.3$ & $2.6-8.3$ \\
GWP data sources & 19 & 18 & 8 & 8 & 13 & 6 & 6 \\
\hline
\end{tabular}

Aluminum, which can be caused by differences in product origin and respective grid energy intensity, production processes included or the time of data recording. We accounted for those variations by compiling Equation 1 with samples drawn from a triangular distribution of the presented GWP ranges. The triangular distribution was therein set up by the minimums and maximums in Table 2 as well as the average over the number of data sources. The number of samples was 10,000. Of course, other materials than the ones listed will be found in eVTOL designs. However, by covering those materials which either have large fractions of the total mass or high GWP emission indices and by including statistical variations of the latter, effects from excluded materials are assumed to be negligible. We chose the functional unit for the CTG analysis to be [1/eVTOL], as referring to 'one instance' of a system is used in LCA studies of aircraft and cars alike [11, 18].

To quantify the sustainability of eVTOL in operation, we applied a Well-to-Shaft analysis (WTS), a form of the Well-to-Wheel (WTW) analysis, which is often used to compare electric and combustion-powered cars in the literature [23]. Equation 2] depicts the WTS scheme and describes the compilation of the impact associated with generation, transport, and consumption of electric power. The WTS analysis thus yields GHG emissions per kWh shaft energy for grid carbon intensity $E I_{\text {grid }}$, drive train losses due to battery charging, discharging and electric motor inefficiencies $E I_{\text {loss }}$, and battery production $E I_{\text {battery. }}$. Typically, the battery production is accounted for in a CTG analysis. In the case of eVTOL, a comparably long operational life of the eVTOL system and limited cycle durability of the battery require batteries to be replaced multiple times. One battery cycle is associated with the electric energy consumed in operation, thus each $\mathrm{kWh}$ of provided shaft power has a share of GHG impact in battery production. The proportion of that share per kWh is determined by the number of battery cycles until replacement $n_{\text {cycles }}$ and the depth of discharge $f_{D o D}$. As Ellingsen et al. point out, the possible battery cycle count can be severely increased by reducing the depth of discharge [24]. Implementing this direct relation is not done in this work and is thus subject to later studies.

$$
E I_{W T S}=E I_{\text {grid }}+E I_{\text {loss }}(P)+E I_{\text {battery }}=E I_{\text {grid }} \cdot\left(1+\frac{1}{\eta(P)}\right)+E I_{\text {battery,prod. }} \cdot \frac{1}{n_{\text {cycles }} \cdot f_{\text {DoD }}}
$$

Finding appropriate values for the grid carbon intensity $E I_{\text {grid }}$ is as important as it is sensitive to the choice of the system boundary. Data provided by states and energy providers may not be as comprehensive as required for LCA. Turconi, Boldrin, and Astrup stress the importance of including impacts of plant operation and infrastructure [25]. They provide statistical data on GHG emissions associated with different power sources, based on a variety of available studies. Together with data provided by the International Energy Association and the World Bank on the electricity generation mix in various countries (2014), collected by Woo and Choi [23], values for grid carbon intensity by country can be calculated. Moro and Lonza further extend the system boundary, as they include impacts from upstream activities and energy trade for the European Union 28 member states (2013) [26]. For this study, we use the data presented by Woo and Choi in order to have a broad statistical basis. Thus, we are not restricted to European data and avoid the possible effect of energy trade. For our study, we chose the United States, as the country is expected to be a pivotal market for UAM [7]. The reported grid energy intensity for the U.S. ranges from 371 to $846 \mathrm{~g} \mathrm{CO}_{2} \mathrm{eq} / \mathrm{kWh}$, with the median and average at 524 and $532 \mathrm{~g} \mathrm{CO}_{2} \mathrm{eq} / \mathrm{kWh}$, respectively. As an example of a potential future energy mix, we use respective data for Canada, which has a high share of hydropower in its energy mix. Canada's reported median and average grid carbon intensity are 177 and $182 \mathrm{~g} \mathrm{CO}_{2} \mathrm{eq} / \mathrm{kWh}$, respectively [23, 25].

As shown in Equation 2, drive train losses are scaled by the grid carbon intensity $E I_{\text {grid }}$ and the efficiency of charging, discharging and the electric motor $\eta(P)$. The total efficiency is summed up from the battery efficiencies [27] 
and the efficiency of the electric motor. The latter is calculated depending on the flight state, based on a parametric model following Barth et al. [28]. The values for GWP in battery production $E I_{\text {battery }}$ strongly depend on the cathode material. For nickel-cobalt-manganese lithium-ion batteries, they range from 120.5 to $172.9 \mathrm{~g} \mathrm{CO}_{2} \mathrm{eq} /\left(\mathrm{kWh}_{\text {Capacity }}\right)$ on a pack level, i. e. including the packaging, battery management system and cell transport [22, 24, 29]. In a pragmatic manner, we assume a maximum cycle count of 750 to 1250, although Ellingsen et al. report, that the possible cycles can be increased to up to $n_{\text {cycles }}=5000$ by limiting the depth of discharge to $f_{D o D}=50 \%$ [24]. According to the Uber Elevate mission requirements, the minimum reserve state of charge is $22 \%$, thus we conduct our analysis with $f_{D o D}=78 \%[8]$.

\section{End of Life considerations}

Of the outlined analyses methods, neither CTG nor WTS takes into account any impacts arising from the end of life (EoL) treatment of eVTOL materials. If included, the possible EoL ways are recycling, incineration, and landfill [21]. Omitting EoL implications was considered valid for the following reasons: First, as we analyzed preliminary to performing this study, the carbon impact of material incineration is by one order of magnitude smaller than the carbon impact of material production, according to emission indices published by Ashby [21]. As will be shown later on, the impact of material production itself is by orders of magnitude smaller than the impact of operation for the majority of the operational life. The role of incineration is thus negligible compared to the carbon impact in actual operation. Secondly and regarding the EoL treatment of batteries, keeping the EoL out of the system boundary is in line with preceding studies, which we use as data sources for the battery LCA [22, 24, 30]. Zackrisson et al. only account for emissions arising from the transport of used batteries to the recycling scrap yard, and further mention, that environmental burdens and benefits from recycling should be allocated to the next product life cycle [22]. Accordingly, Majeau-Bettez et al. mention, that excluding the battery recycling impacts corresponds to a worst-case scenario, as no beneficial secondary material use is allocated to the batteries life cycle [30].

\section{System Sensitivities}

The comparison of WTS analysis of eVTOL operation with ground-based cars requires a meaningful functional unit. In line with the literature, we chose the functional unit to be the actual transport of passengers over a distance. We denote this functional unit by $[-/ \mathrm{P} \mathrm{km}]$; the GHG emission index (EI) in operation is thus measured in $\left[\mathrm{kg} \mathrm{CO} \mathrm{CO}_{2} \mathrm{eq} / \mathrm{P} \mathrm{km}\right]$. Equation 3 provides a simple formulation of the compilation of WTS indices with the actual power requirement $P_{r e q}$, the flight speed $V$, and the number of passengers $n_{P A X}$. The parameter $f_{\text {dist }}$ describes a possible distance saving of air travel compared to ground travel. It thus accounts for a key advantage of eVTOL over ground-based cars. If the travel distance by eVTOL is e.g. only $80 \%$ of the road-bound distance, $f_{c u t}=0.8$. This factor only comes into play when comparing eVTOL with cars or other ground-based means of transport. In a comparative assessment of eVTOL concepts, $f_{\text {cut }}=1.0$.

$$
E I_{\text {operation }}=E I_{W T S} \cdot P_{\text {req }} \cdot \frac{f_{\text {dist }}}{V \cdot n_{P A X}}
$$

As pointed out by Kasliwal et al., mission distance plays a crucial role when assessing the sustainability of eVTOL [9]. We account for this influence in a more general fashion by including the actual hover share, denoted by $f_{\text {hov }}$ as outlined in Equation 4 The hover share is defined by the relative time spent in hover during a mission, thus $f_{\text {hov }}=t_{\text {hov }} / t_{\text {mission }}$. The power required is depending on the concept itself and the flight state, i. e. the flight speed and atmospheric conditions. For the analysis, we use the flight states hover and cruise. The latter can either be defined as the best range flight speed $V_{b R}$, or as the minimum specific GWP flight speed. As will be shown in the results, those velocities only differ slightly due to the power-dependent drive train loss calculation. Thus, both velocities can be used in the analysis. The emission indices and required powers are accordingly labeled by the subscripts hov and crs.

$$
E I_{\text {operation }}=\left(E I_{W T S, \text { hov }} \cdot P_{\text {hov }} \cdot \frac{f_{\text {hov }}}{1-f_{\text {hov }}}+E I_{W T S, \text { crs }} \cdot P_{\text {crs }}\right) \cdot \frac{f_{\text {dist }}}{V_{\text {crs }} \cdot n_{P A X}}
$$

In the sensitivity analyses we alternated the examined quantities in linearly spaced ranges, but not by applying statistical variations as done in the previous calculations. To isolate the effect of each factor, only one factor was varied at a time, while the others were kept fixed at a baseline value. The ranges for those values were $n_{\text {cycles }}=[500,1000,1500,2000], f_{\text {Dist }}=[0.4,0.6,0.8,1.0]$ and $f_{\text {util }}=[3,4,5,6]$. Values for the grid carbon intensity were $E I_{\text {grid }}=\left[177,350,524 \mathrm{~g} \mathrm{CO}_{2} \mathrm{eq} / \mathrm{kWh}\right]$, representing a Canadian and U.S. energy mix as minimum and maximum, and the mean value. 


\section{Results}

\section{Life Cycle Assessment of Urban Air Mobility Concepts}

Figure 2 depicts the GWP associated with the production and operation of an eVTOL on the left and right side, respectively. Note how the functional units differ in the two graphs: While the GWP from production refers to one eVTOL unit produced, the GWP from operation refers to total shaft power provided by the eVTOL. Mind that this segmentation allocates the GWP of battery production to the operation phase, as has been described above. With the included quantification of the uncertainties in input variables, the bars denote the expectation value, while error bars represent the $95 \%$ confidence interval.

\section{Cradle-to-Gate analysis}

The CTG analysis (Figure 2 left) contains results for the three UAM concepts in scope of the study. With a mean GWP of around 24 tons $\mathrm{CO}_{2}$ eq / eVTOL, the Lift+Cruise configuration has the highest carbon impact, followed by the Quadrotor and the Side-by-Side with 19 tons and 17.5 tons, respectively. This order is in line with the empty weights of the three concepts presented in Table 1 . Confidence intervals overlap for the Lift+Cruise and the Quadrotor, as well as for the Quadrotor and the Side-by-Side. However, while material emission indices are represented by distributions based on literature values, material fractions of components remain fixed. Thus, the overlap of confidence intervals is unlikely to allow a change of the shown order. As soon as quantitative estimates on the uncertainty within material shares in components are made, the model can easily be extended accordingly. With that extension, the mentioned overlap of error bars would gain more relevance. The total GWP is mostly caused by the aircraft structure and rotor or propeller mass, contributing around 70 to $80 \%$ of carbon emissions. As mentioned, mind that the component group Propulsion Unit does not contain impacts from battery production, as this is accounted for in the WTS analysis. Including the battery production impact in the CTG analysis increases the GWP of the Propulsion Unit group to the same order of magnitude as the Structure \& Propellers group.
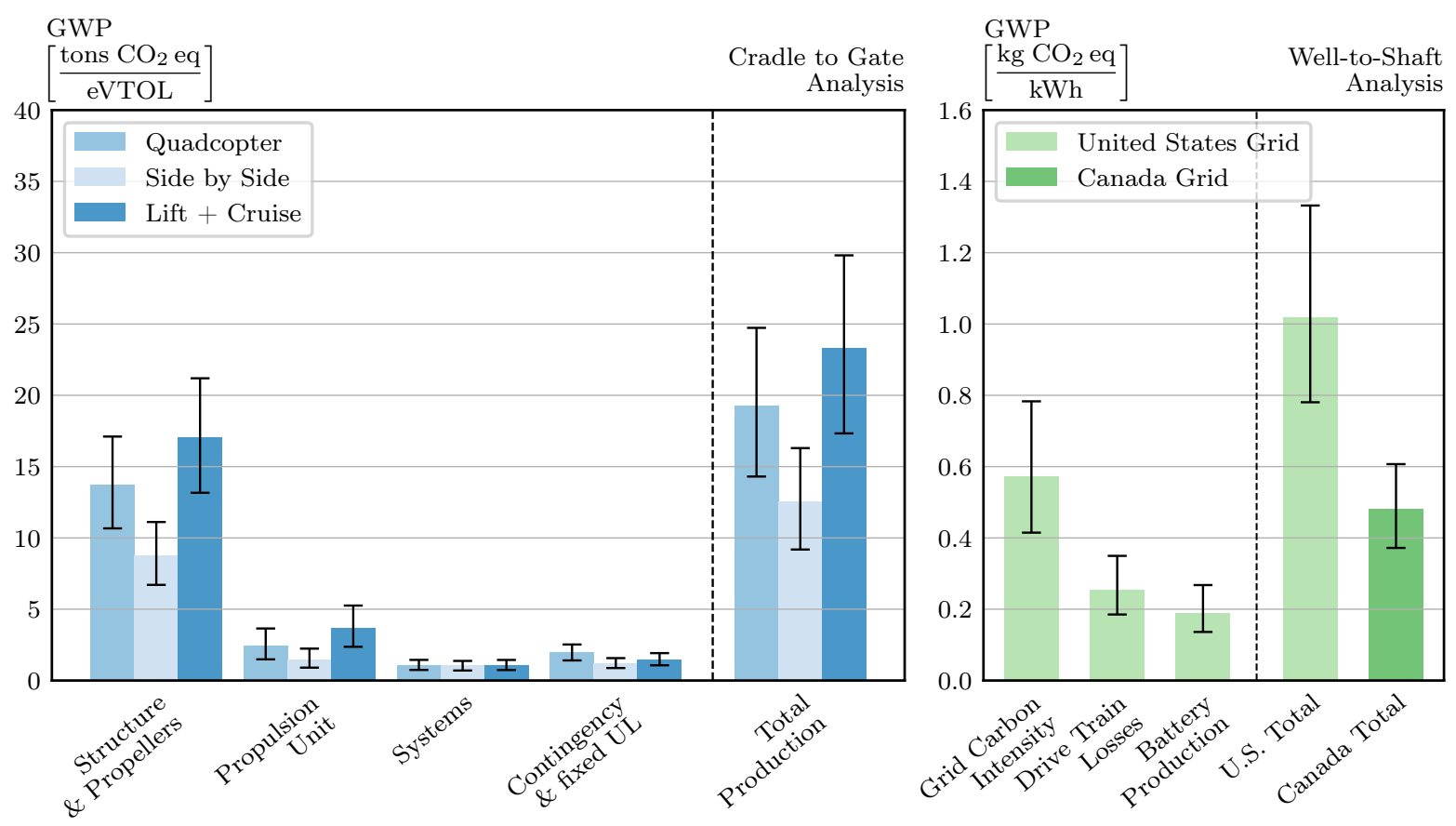

Fig. 2 GHG Emissions due to the production of materials (left) and due to the consumption of electric energy during operation (right). Bars represent mean values. Error bars represent the $95 \%$ confidence interval.

\section{Well-to-Shaft analysis}

The WTS analysis of the operation phase is depicted by the graph on the right. In contrast to the CTG analysis, it is only depicted for the Side-by-Side case. Nevertheless, it is representative for the other concepts, as deviations among the concepts only occur in drive train losses and are smaller than the respective model uncertainties. The first three bars 
show the GWP associated with generation, transport, and use of electric power. The fourth and fifth bar show the total specific GWP for a United States energy grid, and, as an example for a high share in hydropower, a Canadian energy mix, respectively. Among the three contributors, the grid carbon intensity accounts for the majority of impact to the total specific GWP in the case of the U.S. energy grid. With the median and average at 524 and $532 \mathrm{~g} \mathrm{CO}_{2} \mathrm{eq} / \mathrm{kWh}$ as presented in the methods section, the grid carbon intensity contribution is almost double to the share of drive train losses, and three times the share of battery production. Mind that, as outlined before, the losses are directly scaled by the grid carbon intensity. Fewer carbon emissions in primary energy production thus reduce the impact factor of drive train losses. The underlying model for drive train losses is fairly simple and the superposition of uncertainties from grid carbon intensity and from drive train efficiency amplifies the confidence interval range. In contrast, the impact of battery production is quite accurately determined through previous studies and remains constant for variations in grid carbon intensity. In future scenarios with a high share of clean energy production, the battery production may however account for a major share of impact, as a comparison with the Canadian specific total GWP in the rightmost bar shows.

\section{Impacts of Cradle-to-Gate and Well-to-Shaft analyses}

Figure 3 provides a comparison of the impacts of eVTOL production and operation. The total impact from the CTG analysis is divided by the hours of operation, the impact from WTS analysis is multiplied by the required power in cruise flight; the two impacts are thus comparable over the operational lifetime by their now common functional unit, one hour of operation. The scaled impacts from the CTG and WTS analyses are plotted over the operational lifetime on the $x$-axis of Figure 3 and are denoted in $\left[\mathrm{kg} \mathrm{CO}_{2} \mathrm{eq} / \mathrm{h}\right]$. Both axes are logarithmically scaled and thus show the relative importance of impact by orders of magnitude. The specific impact of energy production remains constant during operational life, assuming the grid carbon intensity remains unchanged. Thus, the impacts from eVTOL operation, which are denoted by the green lines, stay level throughout the graph. In contrast, the impacts from production are identical to the total GWP bars in Figure 2 in the first hour of operation and hyperbolically decline thereafter. For the
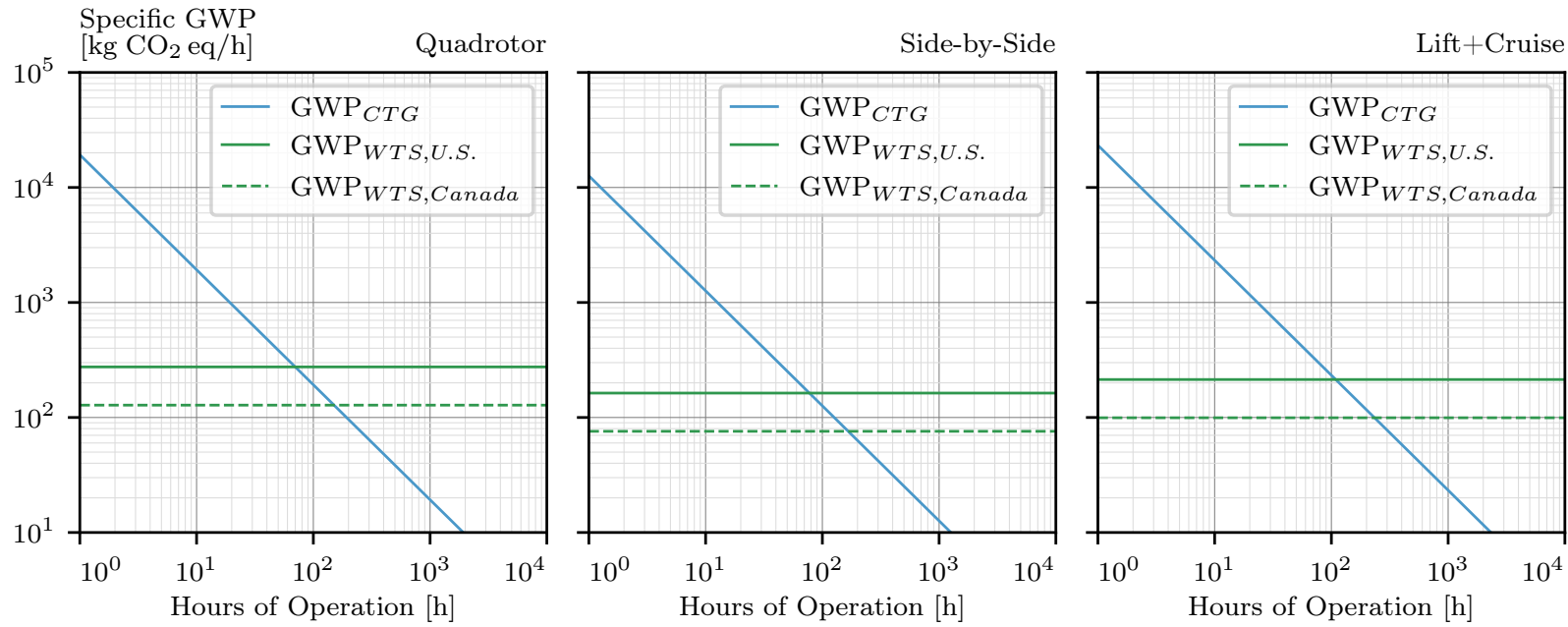

Fig. 3 Comparison of time-specific GWP of material production (based on CTG analysis, depicted by blue lines) and energy consumption in operations (based on WTS analysis, depicted by green lines) for the three eVTOL concepts.

Quadrotor, the Side-by-Side and the Lift+Cruise, the impact of operating the concepts based on a U.S. energy grid becomes equally relevant to the production impact after only 80, 100 and 105 hours of operation, respectively. In a Canadian, less carbon-intense energy grid, these break evens are reached after 108, 110 and 120 hours of operation. For both the examined carbon grid intensities, operational impacts become by orders of magnitude more relevant after only 1000 to 2000 hours of operation. Adding up the values of production and operation leads to the total impact. Not only is it evident, that the relative importance of impacts from production and operation changes quite early in operational life. Furthermore the sustainability ranking of the eVTOL concepts among themselves shifts. Through the production and the early operational life, the Lift+Cruise configuration has the highest carbon impact, followed by the Quadrotor and Side-by-Side concept. As described, this ranking is mainly determined by system empty weight. With the changes of 
life cycle phase impacts after around 80 hours of operation, the Quadrotor leads to the highest carbon impact, due to its high power requirement in forward flight. Although the Lift+Cruise configuration is by far the heaviest of the presented eVTOL concepts, its ability to fly like an airplane above a certain velocity dramatically reduces the power demand, as the lifter propellers no longer require power. In airplane mode the Lift+Cruise concept however still requires more power than the fairly lightweight Side-by-Side concept, which ranks lowest in carbon impact throughout the production and operation phase.

\section{Emission dependence on Power Demand in Forward Flight}

The preceding results section showed how the carbon impact of UAM is dominated by the power demand of eVTOL operation. Figure 3 depicted, that the specific GWP with respect to hours of operation differs among the concepts. This section extends this finding, firstly by introducing the specific GWP with respect to the distance covered, and secondly by depicting the forward flight dependence of the specific GWP. For the three concepts in scope, Figure 4 shows the specific global warming potential (GWP) and power demand on the left and right $y$-axis, respectively, over the envelope of forward flight speeds at $6000 \mathrm{ft}, \mathrm{MSL}, \mathrm{ISA}$. Powers are denoted by blue dots and the mean specific GWP (EV) is denoted by green lines. The light green areas mark the 95\% confidence interval (CI) of the specific GWP. Again, inputs for grid carbon intensity, battery manufacturing impact and possible cycles of the batteries are subject to
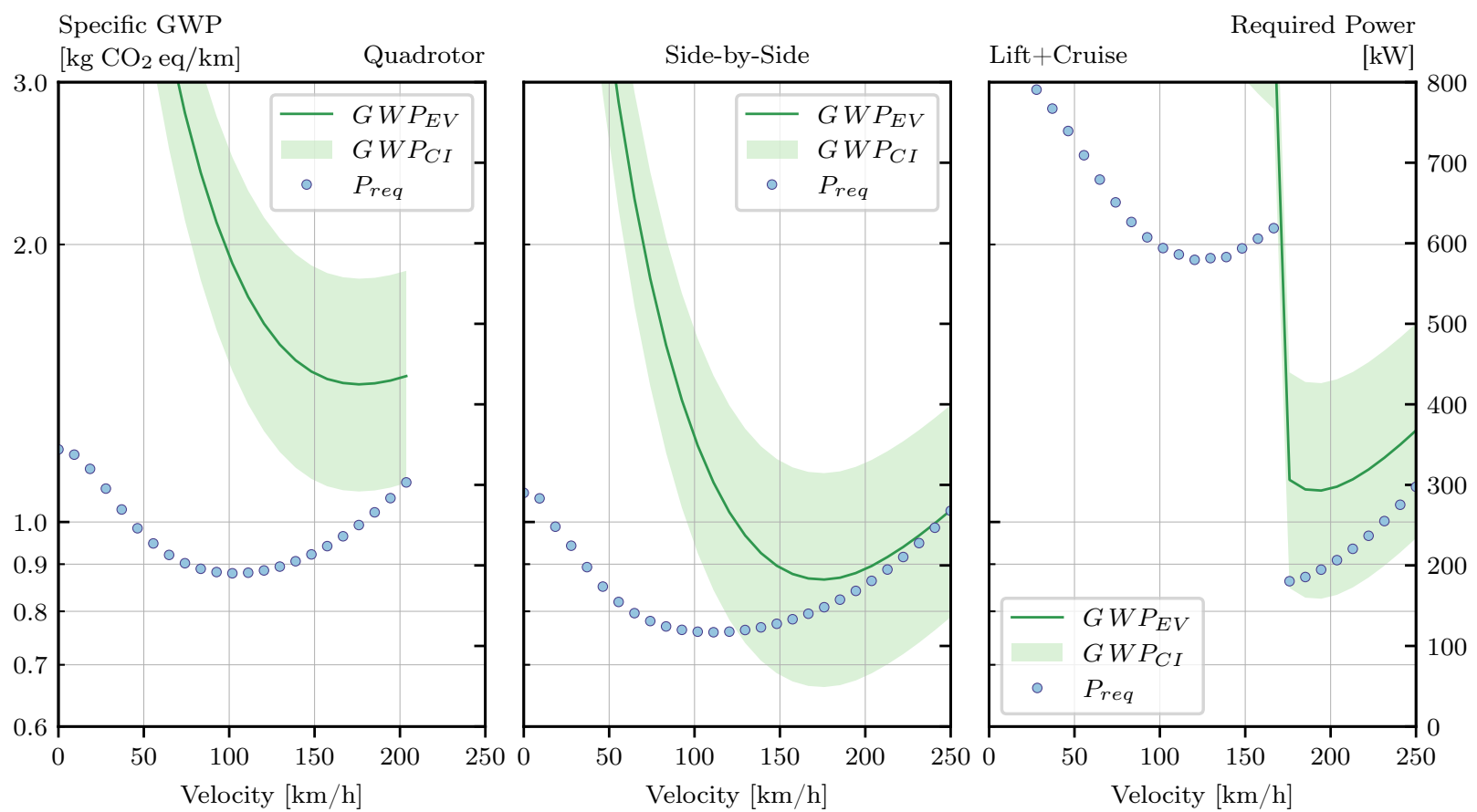

Fig. 4 Powers (blue dots) and distance-specific emissions (green lines) over forward flight speed for the three eVTOL concepts. Emissions include uncertainties in emission indeces of energy production, battery production, and battery cycle durability.

uncertainties. The specific GWP is expressed in relation to distance traveled, thus the unit is $\left[\mathrm{kg} \mathrm{CO}_{2} \mathrm{eq} / \mathrm{km}\right]$. It is plotted on the left $y$-axis of the graphs in a logarithmic scale, while the powers are plotted on a linear scale, yielding the known power curve shape of VTOL aircraft. As discussed in the previous section, Figure 4 shows the dependence of specific GWP and required power to the aircraft weight, now for a range of forward flight speeds. Of the three concepts, the Side-by-Side configuration has the lowest power requirement and lowest GWP per traveled kilometer. The minimum specific GWP occurs approximately at the velocity of best range. Results for the Lift+Cruise configuration mark a large shift in required power and specific GWP at around $170 \mathrm{~km} / \mathrm{h}$. This is due to the changed operation mode of the concept: Below the transition, the concept is assumed to fly in helicopter mode with lifter propellers activated. Above the critical velocity, wings yield sufficient lift and propellers are stopped, leading to a dramatically reduced power requirement. However, the Lift+Cruise configuration indicates only the second-lowest specific GWP. 


\section{Sensitivities: How Sustainable may Urban Air Mobility become?}

This section will give insight to the expectable sustainability of eVTOL in comparison with ground-based cars. We provide insights on the most influential factors that determine the concept's GHG impact with respect to the distance traveled and to the number of passengers transported. Those influential factors are the relative time spent in hover flight, the battery life cycles, the grid carbon intensity, the potential distance saving compared to a ground-based vehicle on the same mission, and the seat utilization. The factors were introduced in in Equation 3 . Accounting for the distinct difference between hovering and forward flight, Equation 4 complemented the hover share as another key factor. We start this section with an easy-to-grasp depiction of grid carbon intensity and the hover share dependence for the three concepts, comparing them the internal combustion engine vehicles (ICEV) and battery electric vehicles (BEV). Then we extend this analysis by varying the most influential factors across expected ranges.

\section{Relevance of mission design and region of operation}

Kasliwal et al. showed that the length of a mission is critical when assessing the sustainability of eVTOL in comparison to ground-based cars [9]. Figure 5 shows the specific GWP over the hover shares in flight states for the three concepts, thus confirming this finding in a more general fashion. While $f_{H o v}=0.0$ represents the rather academic case of a pure forward flight scenario, an increase in $f_{H o v}$ can imply either a shorter mission length at constant hover time or a longer hover time at constant mission length. Extreme cases of missions with more than 50\% hover share, such as urban rescue, are not depicted. The mean GWP is depicted by green solid and green dashed lines for carbon grid intensities of the U.S. and Canada, respectively. Again, the green areas visualize the inherent uncertainty. The grey line and area represent the GWP for a luxury segment battery-powered vehicle (BEV), operated with a North American grid. The red dashed line shows the GWP for a luxury segment internal combustion engine vehicle (ICEV). Both the BEV and the ICEV data are taken from Woo and Choi [23] and for both, the results are divided by 1.54, which is the average number of passengers in car [9]. For this graph, the seat utilization and relative mission length compared to a ground-based commute remain unchanged at $n_{P A X}=6$ and $f_{\text {dist }}=0.8$, thus representing a maximum payload of 6 passengers and a distance reduction of $20 \%$ due to air transport along a more direct line.
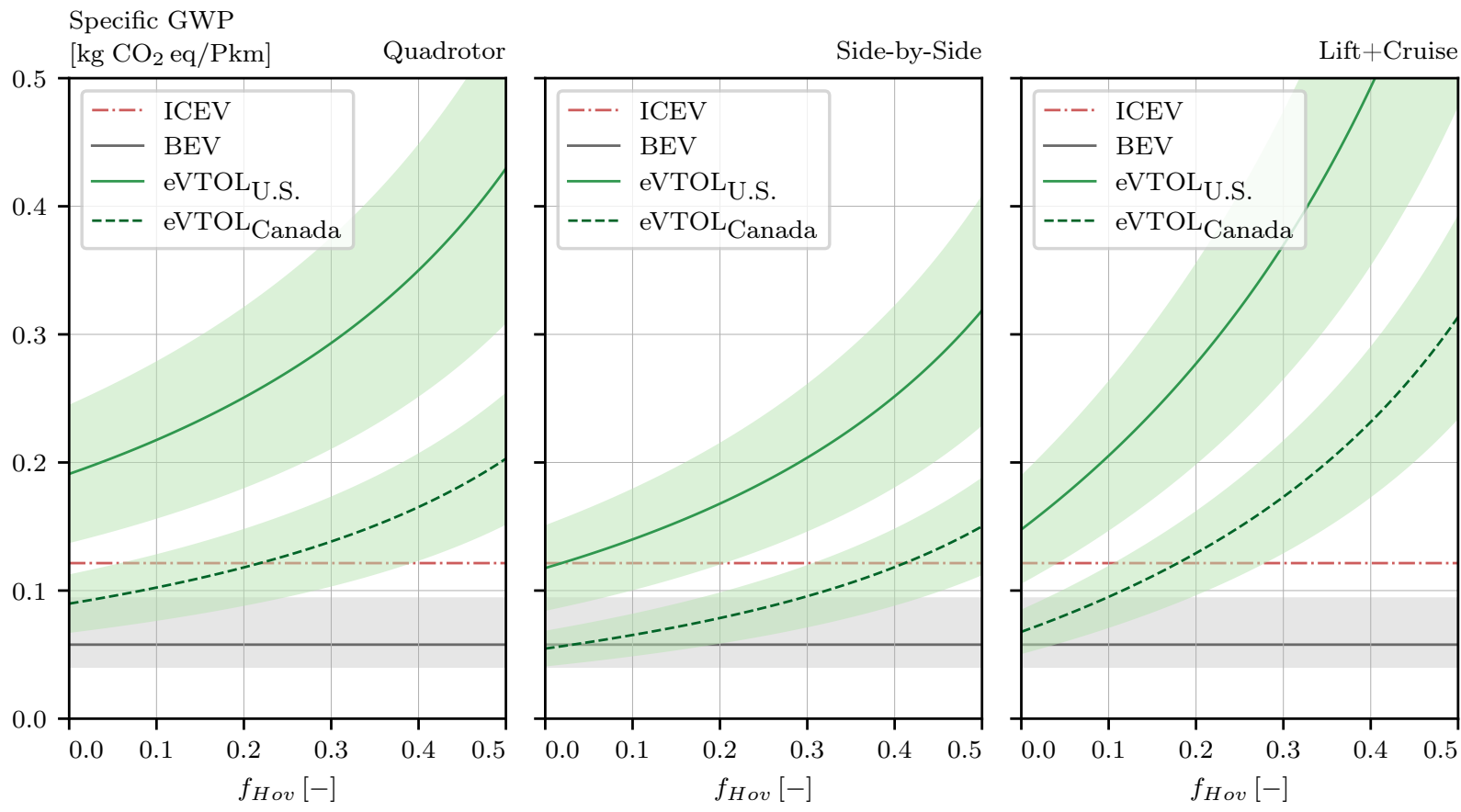

Fig. 5 Specific carbon emissions over the mission hover share, based on a U.S. and Canadian grid carbon intensity, for the three eVTOL concepts. Emissions include uncertainties in emission indeces of energy production, battery production, and battery cycle durability.

Figure 5 shows a strong dependence of the specific GWP on the hover share, which is increasing for higher values of $f_{\text {Hov }}$. In a concept comparison, the Side-by-Side concept shows the smallest GWP over the depicted hover range. 
The Lift+Cruise aircraft ranks second for comparably small hover shares of approximately $f_{\text {Hov }} \leq 0.15$ but shows a high increase of GWP with more time in hover. Mind the respective power curve and the two distinct flight modes of the Lift+Cruise concept, with the compound mode requiring more than twice the power of the airplane mode. Accordingly, the Quadrotor has the highest operational GWP for missions with a low hover share but ranks in the middle for more hover intense missions. In comparison with the operational impacts of ground-based cars, the eVTOL concepts powered by a Canadian energy grid undercut ICEV impacts for a low hover share, i.e. for sufficiently long-ranged missions. A direct comparison of the mean values of the eVTOL concepts and the BEV should be avoided, as the presented values for the BEV are based on a North America carbon grid intensity, including both the U.S. and Canada.

\section{Critical enablers for sustainable UAM operation}

With respect to sustainability we identify the Side-by-Side configuration to be the most promising among the three concepts. Thus we focus the sensitivity study on the Side-by-Side concept. Figure 6 depicts the sensitivities of the eVTOL carbon impact on the most influential factors in scope of this study. Those factors are the possible number of battery charging cycles, the grid carbon intensity, the potential distance saving compared to a ground-based mission, and seat utilization. Input values for each factor are varied around a baseline value. Each subplot in Figure 6 presents the resulting specific GWP for the variation of one of those factors, while the other factors remain at their baseline value. This isolates and identifies the criticality of each factor. The baseline values are $n_{\text {cycles }}=1000, f_{\text {Dist }}=0.8$ and $n_{P A X}=6$. The baseline grid carbon intensity is $E I_{\text {grid }}=177 \mathrm{~g} \mathrm{CO}_{2} \mathrm{eq} / \mathrm{h}$ and represents the average Canadian energy mix. As in Figure 5, the impact is dependent on the hover share and is thus plotted over $f_{H o v}$ on the $x$-axis. The array of curves is colored and marked according to the offset from the baseline, where the baseline case is represented by a dashed green line. Markers and line colors represent offsets from the baseline, with a darker color marking beneficial offsets, and a lighter color marking detrimental offsets. As described before, a direct comparison of the eVTOL curves

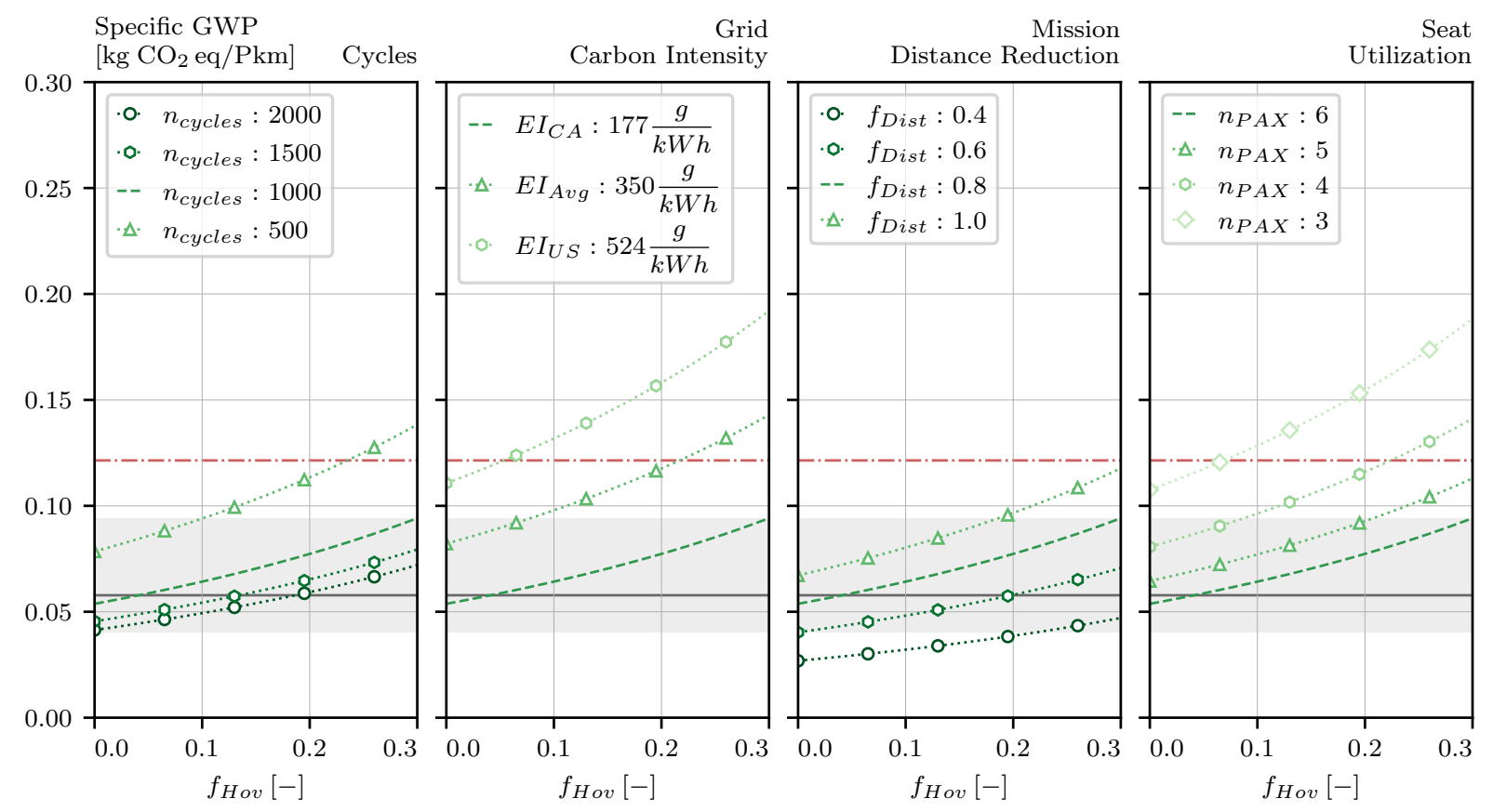

Fig. 6 Sensitivities of key factors on the specific GWP. Baseline values for eVTOL are marked with a dashed line and refer to the average Canadian energy mix. Results for BEV refer to an average North American energy mix.

with the BEV area should be avoided, as the grid carbon intensity behind the BEV results represents a blended North American mix, in contrast to the Canadian energy mix depicted with the eVTOL baseline. However, the grey area shows the possible range of BEV results, including those that are based on a Canadian grid, which we assume to reside within the lower part of the grey range. Two types of GWP sensitivity on the factors can be identified: For the number of battery cycles $n_{\text {cycles }}$ and the seat utilization $n_{P A X}$ we see a hyperbolic decline of specific GWP with increasing factor 
value, which also becomes apparent in Equations 3 and 2 For the grid carbon intensity $E I_{\text {grid }}$ and the mission distance reduction $f_{D i s t}$, the decline appears to be rather linear. As Equation 2 shows, the grid carbon intensity, however, has no linear influence, but is quite dominant on $E I_{W T S}$, particularly for a large battery cycle count. Starting at the baseline result with the Canadian grid energy mix $\left(E I_{\text {grid }}=177 \mathrm{~g} \mathrm{CO}_{2} \mathrm{eq} / \mathrm{h}\right)$ and a fully loaded eVTOL with $n_{P A X}=6$, we identify two factor variations that lead to an eVTOL specific GWP in the lower BEV area: First, an increase of the battery cycle count to $n_{\text {cycles }}$ and second, a further reduction of the mission distance compared to a road-bound commute to $f_{\text {Dist }}$. As the cycle count dependence is hyperbolical, a further increase of $n_{\text {cycles }}$ yields ever-smaller improvements in specific GWP. In contrast, a shorter mission distance has a significant influence on the resulting specific GWP. The actual distance saving is dependent on the specific mission, including regional considerations like road congestion and flight corridors.

\section{Discussion}

We determine the sustainability of eVTOL operation in Urban Air Mobility in terms of greenhouse gas emissions. We show that eVTOL concepts are able to environmentally compete with internal combustion engine vehicles (ICEV) if the required energy is provided by a sufficiently clean energy grid and the UAM mission profile has a small hover share (Figure 5). The environmental potential of battery electric vehicles (BEV) may be met by eVTOL in the following scenario: The provided energy comes largely from renewable energy sources, the eVTOL seats are utilized to a maximum, the UAM mission profile implies a distance saving compared to road-bound mission, and batteries are rechargeable for at least 1000 cycles. We discuss each of those premises in the following:

- Grid carbon intensity: The projection of future grid carbon intensities is not part of this work. However, studies by the German Federal Environment Agency, and by Mai et al. predict a substantial growth of energy demand, also caused by transportation electrification, which may prolong the dependence on fossil energy production [18, 31]. The projected introduction of eVTOL as an additional means of transport could further increase this trend. Therefore, the assumption of the Canadian energy grid for eVTOL operation in this work shall illustrate the potential scenario, rather than predict the general grid carbon intensity at the time of eVTOL market entry.

- Seat utilization: The baseline seat utilization in our sensitivity study in Figure 6 was the maximum concept's payload, $n_{P A X}=6$. This implies that eVTOL will not require a pilot for operation. To date, it is however not clear, if and when fully autonomous flight will be possible. Thus, a near term environmental assessment of UAM would require a limitation to a maximum of $n_{P A X}=5$, which in turn increases the passenger-specific impact.

- Urban Air Mobility mission profile: To be environmentally competitive, UAM missions need to be rather longranged, require a preferably short hover time, and save as much distance as possible compared to ground-based cars. A short case example of a standard UAM mission may illustrate this: The commute from New York JFK Airport to Manhatten along the Grand Central Parkway measures approximately 16mi. The direct distance is around $12.5 \mathrm{mi}$, thus the saving is $f_{\text {Dist }}=0.78$. By car, this commute can take over an hour, compared to an air taxi hop with the Side-by-Side configuration at $V_{b R}$ of $7.1 \mathrm{~min}$. We assume a hover time of $90 \mathrm{sec}$, leading to a hover share of $f_{H o v}=0.21$. With a maximum seat utilization and the introduced carbon grid intensities for the U.S. and Canada, the respective specific GWP of this mission would be around 160 and $80 \mathrm{~g} \mathrm{CO}_{2} \mathrm{eq} / \mathrm{Pkm}$ ('per passenger-kilometer'), compared to the BEV's impact of $58 \mathrm{~g} \mathrm{CO}_{2} \mathrm{eq} / \mathrm{Pkm}$ for the average 1.54 passengers.

- Battery technology: All calculations in this study assume an effective energy density of the Lithium-Ion battery on pack level of $400 \mathrm{Wh} / \mathrm{kg}$, which implies an even higher energy density of around $650 \mathrm{Wh} / \mathrm{kg}$ at a cell-specific level [4]. Silva et al. describe this 'aggressive target' as a valid approach to presume a certain technology readiness level, which is required to realize UAM at 'reasonable size and cost' [4]. However, lower energy density eVTOL vehicles with a heavier battery and a rather confined reference mission may be introduced in near future. Such vehicles would require more power and thus lead to a higher specific impact than the concepts presented here.

We extend the finding of Kasliwal et al. who recently reported lower emissions for highly utilized eVTOL in an article in 'nature Communications' [9]. However, we report a more conservative estimation of UAM impacts due to the following differences regarding modeling and data: First, our operational emissions formulation and the consistent aircraft performance model imply variations. Battery production emissions account for a third of the impact per $\mathrm{kWh}$ and including them leads to a higher total impact. Our power-dependent power train efficiency leads to efficiencies of $\eta=0.7-0.8$, which is more conservative than the battery efficiency of 0.9 used by Kasliwal et al. [9]. The consistent aircraft modeling and performance computation, that comes with the application of NDARC and the eVTOL concepts sized by Silva et al. and Johnson et al., also yields a more conservative set of design parameters [3, 4]. While Kasliwal et al. set their base-case lift-to-drag $(L / D)$ ratio to 17 , according to Uber, our concepts $L / D$ ratios range from 5.8 to 8.5 
[4, 8, 9]. According to Datta et al. maximum $L / D$ ratios are up to approximately 5, 8 and 9 for compound helicopters, tilt-rotors and propeller aircraft, respectively [32]. Second, and as for differences in data, Kasliwal et al. assume the U.S. grid carbon intensity to be fixed at $486 \mathrm{~g} \mathrm{CO}_{2} \mathrm{eq} / \mathrm{kWh}$ and a cruise altitude of $h=1000 \mathrm{ft}$, while we use a sampled distribution with a mean value of $532 \mathrm{~g} \mathrm{CO}_{2} \mathrm{eq} / \mathrm{kWh}[9]$ and $h=6000 \mathrm{ft}$. Similar or equal assumptions compared to Kasliwal et al. are the specific battery energy $(400 \mathrm{Wh} / \mathrm{kg})$, the battery depth-of-discharge $\left(f_{D o D}=0.78\right.$ to 0.8$)$, the ICAV and BEV utilization of $f_{\text {util,car }}=1.54$, as well as comparable values for empty weight ratio and disk loading [9]. However, we do not include any power implications of axial flight, which would further increase the calculated emissions, and thus will be implemented in the future.

As for further results, we depict design implications of eVTOL concepts over their flight envelope (Figure 4 . Unsurprisingly, a minimum empty weight ratio and a maximum battery energy density are desired, as both have a direct implication on the eVTOL weight and induced power. For the Quadrotor and the Side-by-Side, for which $E W / D G W=0.5$ and 0.49 , respectively, the power required in low speed is fairly comparable, as depicted in Figure 4 The Lift+Cruise configuration with $E W / D G W=0.59$ and a large number of propellers requires more than double the power in hover and low speed. Also, aircraft drag indicates a major influence. In fast forward flight, the Quadrotor is not only limited to a maximum cruise speed of $200 \mathrm{~km} / \mathrm{h}$ but also has a distinctly higher power requirement and specific emission than the Side-by-Side helicopter. For the two concepts, the equivalent drag area is $1.2 \mathrm{~m}^{2}$ and $0.7 \mathrm{~m}^{2}$, respectively. The Lift+Cruise concept, with a drag of $1.57 \mathrm{~m}^{2}$ in airplane mode and the resulting high power and emissions, displays the importance of aerodynamically lean designs.

We provide a comparison of greenhouse gas impacts from eVTOL production and from eVTOL operation (Figure 3). Although eVTOL systems will be fabricated with a large fraction of carbon fiber reinforced plastic, a material that leads to high carbon emissions in production due to excessive energy requirements, we show that the overall production impact is most likely negligible. Even with a comparably clean grid energy mix, the carbon impact from eVTOL operation dominates its total footprint after a few hundred hours of operation. Regarding the use of bio-based materials in aircraft structures, Strohrmann et al. come to a similar conclusion, as they show that the emission reduction potential of bio-based materials is significantly restricted by their weight implications [10].

\section{Conclusion}

Our study provides comprehensive insights into the expected sustainability of Urban Air Mobility. We discuss the influence of design parameters on environmental vehicle performance and analyze key enablers for a preferably sustainable role of eVTOL in a future urban mobility. Facilitated by our robust analysis approach we show, that environmental competitiveness of flying cars with conventional cars is unlikely in a current scenario but could be impinged by an optimistic set of external factors together with economic vehicle designs. The key requirements for the sustainable operation of eVTOL aircraft in Urban Air Mobility are a high share of carbon-neutral means of energy production and bold advancements in battery technology. Regarding the ongoing development process towards Urban Air Mobility, engineers, researchers and decision-makers can and should consider the discussed factors and requirements, in order to design the eVTOL technology in a sustainable way.

\section{Acknowledgement}

This work is supported by the German Federal Ministry for Economic Affairs and Energy through the German Aviation Research Program LuFo V-3 in the project EcoDraft.

Supported by:

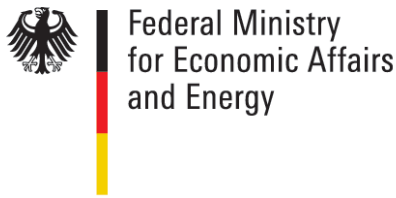

on the basis of a decision

by the German Bundestag 


\section{References}

[1] Whittle, R., “Air Mobility Bonanza Beckons Electric VTOL Developers,” Vertiflite, Mar-Apr, 2017.

[2] Vertical Flight Society Staff, "Electric VTOL News - The Latest in eVTOL Developments,”Vertiflite, May-Jun, 2017.

[3] Johnson, W., Silva, C., and Solis, E., “Concept Vehicles for VTOL Air Taxi Operations," AHS International Technical Meeting on Aeromechanics Design for Transformative Vertical Flight, San Francisco, CA, 2018.

[4] Silva, C., Johnson, W. R., Solis, E., Patterson, M. D., and Antcliff, K. R., "VTOL Urban Air Mobility Concept Vehicles for Technology Development,” 2018 Aviation Technology, Integration, and Operations Conference, 2018, p. 3847.

[5] Patterson, M. D., Antcliff, K. R., and Kohlmann, L. W., "A Proposed Approach to Studying Urban Air Mobility Missions including an Initial Exploration of Mission Requirements," AHS International 74th Annual Forum \& Technology Display, Phoenix, Arizone, Mar-Apr, 2018.

[6] Garrow, L. A., German, B., Mokhtarian, P., Daskilewicz, M., Douthat, T. H., and Binder, R., "If You Fly It, Will Commuters Come? A Survey to Model Demand for eVTOL Urban Air Trips," 2018 Aviation Technology, Integration, and Operations Conference, 2018, p. 2882.

[7] Hasan, S., "Urban Air Mobility (UAM) Market Study,” 2018.

[8] Holden, J., and Goel, N., "Fast-Forwarding to a Future of On-Demand Urban Air Transportation," San Francisco, CA, 2016.

[9] Kasliwal, A., Furbush, N. J., Gawron, J. H., McBride, J. R., Wallington, T. J., De Kleine, R. D., Kim, H. C., and Keoleian, G. A., "Role of flying cars in sustainable mobility," Nature Communications, 2019.

[10] Strohrmann, K., and Hajek, M., "An Eco-Efficient Helicopter Tailplane Hybridized from Flax, Balsa and Carbon,” AIAA SciTech Forum - 55th AIAA Aerospace Sciences Meeting, 2019.

[11] Johanning, A., "Methodik zur Ökobilanzierung im Flugzeugvorentwurf," Ph.D. thesis, Technische Universität München, 2017.

[12] Basset, P.-M., Reboul, G., DangVu, B., and Mercier, S., "Rotorcraft Performance and Environmental Impact Evaluation by Multidisciplinary Modelling," World Academy of Science, Engineering and Technology, International Journal of Mechanical, Aerospace, Industrial, Mechatronic and Manufacturing Engineering, Vol. 11, No. 2, 2017, pp. 284-298.

[13] Finnveden, G., Hauschild, M. Z., Ekvall, T., Guinée, J., Heijungs, R., Hellweg, S., Koehler, A., Pennington, D., and Suh, S., "Recent developments in life cycle assessment," Journal of environmental management, Vol. 91, No. 1, 2009 , pp. 1-21.

[14] Johnson, W., "NDARC-NASA Design and Analysis of Rotorcraft," Tech. rep., National Aeronautics and Space Administration, 2015.

[15] Goedkoop, M., Heijungs, R., Huijbregts, M., De Schryver, A., Struijs, J., and Van Zelm, R., "ReCiPe 2008," A life cycle impact assessment method which comprises harmonised category indicators at the midpoint and the endpoint level, Vol. 1, 2009, pp. $1-126$.

[16] Hauschild, M. Z., Rosenbaum, R. K., and Olsen, S. (eds.), Life Cycle Assessment - Theory and Practice, Springer, 2018.

[17] "Global Warming of $1.5^{\circ} \mathrm{C}$. An IPCC Special Report on the impacts of global warming of $1.5^{\circ} \mathrm{C}$ above pre-industrial levels and related global greenhouse gas emission pathways, in the context of strengthening the global response to the threat of climate change, sustainable development, and efforts to eradicate poverty," Tech. rep., World Meteorological Organization, Geneva, Switzerland, 2018.

[18] Helms, H., Lambrecht, U., Jöhrens, J., Pehnt, M., Liebich, A., Weiß, U., and Kämper, C., "Ökologische Begleitforschung zum Flottenversuch Elektromobilität" Twin Drive,", 2013.

[19] Wernet, G., Bauer, C., Steubing, B., Reinhard, J., Moreno-Ruiz, E., and Weidema, B., "The ecoinvent database version 3 (part I): overview and methodology,", Sep 2016.

[20] Das, S., "Life cycle assessment of carbon fiber-reinforced polymer composites," The International Journal of Life Cycle Assessment, Vol. 16, No. 3, 2011, pp. 268-282.

[21] Ashby, M. F., Materials and the environment: eco-informed material choice, Elsevier, 2012.

[22] Zackrisson, M., Avellán, L., and Orlenius, J., "Life cycle assessment of lithium-ion batteries for plug-in hybrid electric vehicles-Critical issues," Journal of Cleaner Production, Vol. 18, No. 15, 2010, pp. 1519-1529. 
[23] Woo, J., Choi, H., and Ahn, J., "Well-to-wheel analysis of greenhouse gas emissions for electric vehicles based on electricity generation mix: A global perspective," Transportation Research Part D: Transport and Environment, Vol. 51, 2017, pp. 340-350.

[24] Ellingsen, L. A.-W., Majeau-Bettez, G., Singh, B., Srivastava, A. K., Valøen, L. O., and Strømman, A. H., "Life cycle assessment of a lithium-ion battery vehicle pack," Journal of Industrial Ecology, Vol. 18, No. 1, 2014, pp. 113-124.

[25] Turconi, R., Boldrin, A., and Astrup, T., "Life cycle assessment (LCA) of electricity generation technologies: Overview, comparability and limitations," Renewable and sustainable energy reviews, Vol. 28, 2013, pp. 555-565.

[26] Moro, A., and Lonza, L., "Electricity carbon intensity in European Member States: Impacts on GHG emissions of electric vehicles," Transportation Research Part D: Transport and Environment, Vol. 64, 2018, pp. 5-14.

[27] Campanari, S., Manzolini, G., and De la Iglesia, F. G., "Energy analysis of electric vehicles using batteries or fuel cells through well-to-wheel driving cycle simulations," Journal of Power Sources, Vol. 186, No. 2, 2009, pp. 464-477.

[28] Barth, A., Spieß, C., Kondak, K., and Hajek, M., "Design, Analysis and Flight Testing of a High Altitude Synchropter UAV," American Helicopter Society 74th Annual Forum, 2018.

[29] Amarakoon, S., Smith, J., and Segal, B., "Application of life-cycle assessment to nanoscale technology: Lithium-ion batteries for electric vehicles," Tech. rep., 2013.

[30] Majeau-Bettez, G., Hawkins, T. R., and Strømman, A. H., "Life cycle environmental assessment of lithium-ion and nickel metal hydride batteries for plug-in hybrid and battery electric vehicles," Environmental science \& technology, Vol. 45, No. 10, 2011, pp. $4548-4554$.

[31] Mai, T., Steinberg, D., Logan, J., Bielen, D., Eurek, K., and McMillan, C., "An Electrified Future: Initial Scenarios and Future Research for US Energy and Electricity Systems,” IEEE Power and Energy Magazine, Vol. 16, No. 4, 2018 , pp. $34-47$.

[32] Datta, A., Elbers, S., Wakayama, S., Alonso, J., Botero, E., Carter, C., and Martins, F., "Commercial Intra-City On-Demand Electric-VTOL Status of Technology,", 2018. 